\title{
Flywheel Energy Storage Advances Using HTS Bearings
}

Thomas M. Mulcahy, John R. Hull, Kenneth L. Uherka, and Ralph C. Niemann

Energy Technology Division, Argonne National Laboratory, Argonne, IL 60439

Robert G. Abboud, John P. Juna, and John A. Lockwood

Commonwealth Research Corporation, Chicago, IL 60690

August 1998

The submitted manuscript has been created by the University of Chicago as Operator of Argonne National Laboratory ("Argonne") under Contract No. W-31-109-ENG-

38 with the U.S. Department of Energy. The U.S.

Government retains for itself, and others acting on its behalf,

a paid-up. nonexclusive, irrevocable worldwide license in

said article to reproduce, prepare derivative works, distribute

copies to the public, and perform publicly and display

RECEIVED

SEP 211999

OSTI

publichy, by or on behalf of the Government

Distribution

R. W. Weeks

R. B. Poeppel

U. Balachandran

R. A. Valentin

Authors

ESA Section

ET Division File

F. Y. Fradin

H. Drucker

S. Lake

To be presented at 1998 Applied Superconductivity Conference, September 13-18, 1998, Palm Desert, CA.

*Work supported by the U.S. Department of Energy, Energy Efficiency and Renewable Energy, as part of a program to develop electric power technology, under Contract W-31-109-Eng-38. 


\section{DISCLAIMER}

This report was prepared as an account of work sponsored by an agency of the United States Government. Neither the United States Government nor any agency thereof, nor any of their employees, make any warranty, express or implied, or assumes any legal liability or responsibility for the accuracy, completeness, or usefulness of any information, apparatus, product, or process disclosed, or represents that its use would not infringe privately owned rights. Reference herein to any specific commercial product, process, or service by trade name, trademark, manufacturer, or otherwise does not necessarily constitute or imply its endorsement, recommendation, or favoring by the United States Government or any agency thereof. The views and opinions of authors expressed herein do not necessarily state or reflect those of the United States Government or any agency thereof. 


\section{DISCLAIMER}

Portions of this document may be illegible in electronic image products. Images are produced from the best available original document. 


\title{
Flywheel Energy Storage Advances Using HTS Bearings
}

\author{
Thomas M. Mulcahy, John R. Hull, Kenneth L. Uherka, and Ralph C. Niemann \\ Energy Technology Division, Argonne National Laboratory, Argonne, IL 60439 \\ Robert G. Abboud, John P. Juna, and John A. Lockwood \\ Commonwealth Research Corporation, Chicago, IL 60690
}

\begin{abstract}
High-Temperature-Superconducting (HT) bearings have the potential to reduce idling losses and make flywheel energy storage economical. Demonstration of large, high-speed flywheels is key to market penetration. Toward this goal, a flywheel system has been developed and tested with $5-\mathrm{kg}$ to 15 kg disk-shaped rotors. Rim speeds exceeded $400 \mathrm{~m} / \mathrm{s}$ and stored energies were $>\mathbf{8 0} \mathrm{W}-\mathrm{hr}$. Test implementation required technological advances in nearly all aspects of the flywheel system. Features and limitations of the design and tests are discussed, especially those related to achieving additional energy storage.
\end{abstract}

\section{INTRODUCTION}

Commercial viability of diurnal energy storage demands [1] kW-hr rated flywheels, as well as the low losses realized with small, low-speed, HTS bearings [2], [3]. A typical installation would include multiple flywheels. Already, a prototypic-sized permanent-magnet (PM) rotor element, B1, has been designed and fabricated for a high-speed HTS bearing [4]. Tests at low speeds demonstrated low losses. High-speed testing of B1, and two other rotors, B2 and B3, are reported here. Similar world-wide efforts are in progress [5], using different designs .

The goal is to demonstrate operation of an HTS bearing in prototypic diameter rotors at high speeds, but not high energy levels. Increased energy storage is achievable by making longer rotors, for instance. Regardless, this scale-up testing addresses issues that need resolution for prototypic energylevel flywheels, and develops prototypic components: a stator for the HTS bearing, connections for high rim-speed rotors, additional lift bearings, and a large-gap motor/generator.

\section{TEST FACILITY AND COMPONENTS}

The approximately $1-\mathrm{m}$ diameter and height of the cylindrical-shaped vacuum boundary of the test facility [2] was chosen larger than necessary. See Fig. 1. The additional clearances and stainless-steel material of the boundary assured eddy-current losses would be minimal, due to the rotating field of the B1 PM, for instance. Also, additional space eased accommodation and evaluation of components, otherwise inaccessible in a prototypic installation. The cylinder's ends have flanges and O-rings, thus the 64-mm thick bottom plate (Datum) and the 32-mm thick top (Cover) are removable.

Manuscript received September 13, 1998.

This work was supported by the U.S. Department of Energy, Energy Efficiency and Renewable Energy, as part of a program to develop electric power technology, under Contract W-31-109-Eng-38.
Vacuums of $1.3310^{-5} \mathrm{~Pa}\left(10^{-7}\right.$ torr) were achievable with a Leybold 1000 1/s TMP1000/NT20 turbomolecular pump and the added cryopumping of the HTS's LN cryohead. Vacuum measurements and reliable valve operation and sequencing were made with a Granville-Phillips 307 controller, equipped with Convectron (Pirani) and hot filament (Bayard-Alpert) ionization gauges. The Datum was mounted on a massive meter-high base, which was leveled and attached to a quiet concrete floor with elastomeric materials. The base allowed access to the Datum and provided a reaction mass, which, in combination with the low stiffness PM and HTS suspension, adequately isolated the rotor from external excitation.

\section{A. HTS Stator (Cryohead)}

For the levitation and rotation of B1-B3, four LN chambers were built that contains about 70 hexagonal-shaped HTS, arranged in a close-packed array under the $0.127-\mathrm{m} \mathrm{ID}$ and $0.203-\mathrm{m}$ OD of the rotors' PM rings. For the last three arrays, each centrally-seeded, melt-grown HTS was formed in the same die from the same materials, resulting in a nominal height of $\sim 12.5 \mathrm{~mm}$ and a distance across the flats of $\sim 22$ $\mathrm{mm}$. The HTS are from Superconductive Components, Inc., who use a refined stoichiometry and manufacturing process derived from work at Argonne [6]. Quality control of the levitation capabilities of each HTS was monitored. A zerofield-cooled force-hysterisis curve was measured, as a standard $12.5-\mathrm{mm}$ diameter $\mathrm{NdFeB} \mathrm{PM}$ was lowered and raised, and the maximum force at zero height, $F_{0}$, was extrapolated. Measurement was automated, but is the same as performed manually [6]. Early HTS had $\mathrm{F}_{0}<5$, while the latest are $>20$. Each array was formed by symmetric placement of similar HTS and identified with the average $F_{0}$.

Cryohead construction evolved during fabrication. Studies with small arrays and PMs found that uniform height of the top surface of each array and adequate cooling of each HTS were most important in optimizing levitation height. Thus, the array of HTS are placed upside down and in a flat removable annular mold and potted in Epon 828 epoxy. Right after the mold is filled, just above the HTS, an inchthick ring of open-pore alumina foam (Fiber Ceramic, Inc.'s molten-steel filters) is positioned and leveled above the HTS, in the epoxy. The 30-ppi ceramic ring has an open porosity of $85 \%$ and the array's ID and OD, which provide both direct LN flow paths to the bottom of the array and a solid mount.

The array, now attached to the ceramic ring, is mounted inside and just below ( $<1 \mathrm{~mm}$ ) the $3-\mathrm{mm}$ thick cover of a cylindrical G-10 vacuum boundary, machined from a $280-\mathrm{mm}$ diameter tube and flat plates. Elimination of eddy current losses and maximization of levitation height, required electrically non-conductive material and the thinnest cover that could contain the vacuum and $\mathrm{LN}$ pressure $(<276 \mathrm{kPa})$. 
Attachment of the top and bottom plates to the tubular sides was accomplished by threading and gluing, such that joints remain in compression during temperature cycling. Also, surfaces of the G-10 are selectively painted with epoxy to seal machined surfaces, especially those along the grain of the G10 's glass fabric. The G-10 vacuum boundary includes two feedthroughs, to direct $\mathrm{LN}$ below the porous ceramic ring and to vent above the array. The thick bottom plate of the Cryohead enabled rigid attachment to the Datum.

Single-pass LN flow through the Cryohead was by gravity feed from a 2-m higher reservoir, which was automatically filled by LN dewars. Gravity flow was chosen for its quiet operation during energy loss measurements and low cost. Various foam insulation were used. Closed-cycle systems with double-wall piping are envisioned for prototype applications.

\section{B. Three High Rim-Speed Disk Rotors}

The 25.4-mm thick B1 rotor [4] was constructed to sustain $400-\mathrm{m} / \mathrm{s}$ speeds at its $0.4-\mathrm{m}$ OD. Higher rim speeds and energy storage were made possible by adding two largerdiameter filament-wound inertia rings to B1, resulting in the $\mathrm{B} 2$ rotor, with a $0.59-\mathrm{m}$ OD. The smaller AS4 carbon ring added had a $0.467-\mathrm{m}$ OD and was attached to the OD of the $\mathrm{B} 1$ and the ID of the larger AS4 ring by 4-mm radially-thick annuli filled with urethane. B1 filament-winding techniques $[4,7]$ were used in all rotor composite fabrication, and the urethane connections had been developed and proven [7]. The APC/Ethacure 300 urethane and annulus width were chosen to sustain rotor speeds and the strain mismatch between the different composite rings. The maximum rim speed was still limited by B1 to $<600 \mathrm{~m} / \mathrm{s}$. Inertia properties are in Table I.

Opening of the circumferential gaps between the pieshaped ring-magnet segments limits the speed of $B 1$ [4]. This restraint was removed in the design of rotor B3, see Fig. 2, by banding PM the segments with a $62 \%$ stiffer carbon material, Toray's M46J. Otherwise, the geometry of B3 and B1 are the same. B3 is limited to rim speeds $<800 \mathrm{~m} / \mathrm{s}$ by the magnet segments radial crushing their bands. At the highest speeds, B3's PM gaps are predicted to open by $0.5 \mathrm{~mm}$, which were measured to have little effect on the circumferential uniformity of the field experienced by the HTS, at the $>6-\mathrm{mm}$ levitation heights expected. Potting the magnets in urethane prevented magnet pieces from ejecting during rotation.

Other B3 improvements were made without compromise of performance. The magnetic return-path ring, or Shunt, between the inner and outer PM magnetic rings is a maraging steel, Carpenter's Nimark 300, which has a $400 \%$ larger yield stress than Bl's silicon iron. Also, the PM bands were not pressfit, and the outside M46J PM ring was banded separately from the Shunt. Strain incompatibility between the PM and Shunt bands was no longer an issue. Finally, the inertia rings were attached with a urethane annulus, as for B2, because they did not have to constrain the magnet deformation, as in B 1. The inertia section consists of a T700 carbon ring lightly press fit on a $0.32-\mathrm{m}$ OD $\times 0.24-\mathrm{m}$ ID E-glass ring.

\section{Spin-up Motors and Spin-down Generators}

Several motor and generator units, M/Gs, were used and all performed their function, which evolved from studying idling losses to evaluating prototypic designs. All involved synchronize coupling of a stator's rotating magnetic field with passive conductive or PM elements on the rotor.

The unit suited for measuring flywheel idling losses used in-house eddy-current clutches, which are depicted in Fig. 1. The magnetic-clutch torque coupling was very inefficient, but its parallel-disk design accommodated large, lateral misalignments and rotor-critical vibrations that are inherent in low-stiffness HTS/PM suspensions. Also, only small lateral forces develop. Most importantly, the drive disk of the clutch could be benignly retracted after spin-up: no external magnetic fields or conductors were present during loss measurements.

The clutch consisted of two high-strength, 7075-T6, disks: a $6.4-\mathrm{mm}$ thick aluminum disk mounted on the rotor and a disk, with embedded PMs, which was spun at the end of a shaft connected to the external M/G through a vacuum-tight rotary feedthrough. The clutch's PM disks contained 6 or 8 , 9.5-mm diameter and $12.5-\mathrm{mm}$ long, $\mathrm{NdFeB} \mathrm{PMs}$, pressfit into hole circles, which had ODs up to $90 \mathrm{~mm}$. The PMs were arranged with alternating polarity and a steel shunt ring was mounted on the back of the aluminum. The PM-disks design can be optimized to maximize torque [8].

Externally-mounted 1/4-hp and 5-hp American Motion Systems' electroturbines were used with Rigaku medium and high-speed ferrofludic feedthroughs, which had maximum speeds of $15 \mathrm{krpm}$ and $50 \mathrm{krpm}$, respectively. This $\mathrm{M} / \mathrm{G}$ system (ETSC) was limited to $<10 \mathrm{krpm}$, by resonant vibrations of the long shaft connecting the clutch to the feedthroughs. In an attempt to bypass these problems, a set of stator solenoids (Coils) were built to operate in the vacuum and provide the rotating magnetic field to the rotor's clutch plate. See Fig. 3.

The back end of 24 Coils were mounted above the rotor clutch plate, on a ring made of laminated transformer sheets. See Fig. 3. The initial gap between the top of the clutch plate and the bottom of the Coils, equally spaced on a $181-\mathrm{mm}$ diameter, was adjustable over several millimeters. Each 48$\mathrm{mm}$ long Coil was made by wrapping 100 turns of \# 16 copper wire over a $12.5-\mathrm{mm}$ diameter steel tube filled with a ferrite core, resulting in a $22-\mathrm{mm}$ OD. The Coils were powered with 3-phase, each at 110 volts, and could be arranged in different motor configurations. For instance, the third phase for a 4 pole rotating magnetic field used every fifth and sixth coil connected with the same polarity. To decelerate, the motor was run in reverse. At a rotor speed of nearly $10,000 \mathrm{rpm}$, the Coil temperature rose to nearly $125^{\circ} \mathrm{C}$, which effected other components' functions. It was used until the Halbach M/G unit became available.

A di-pole Halbach M/G design [9] was chosen, because large radial gaps are possible, nearly pure torque is produced, with very small lateral forces, the rotor elements are passive PMs, and stray external magnetic fields on the rotor are minimal. It was built for larger prototype flywheels, but it was used with rotor B3 to gain operating experience. A cross section of the Halbach rotor is shown in Fig. 2, mounted in rotor B3 via another urethane-filled annular gap. 
The Halbach PM ring (100-mm OD x 80-mm ID x 55-mm long) was made from 16 UGIMAX $34 \mathrm{~K}$ bar-like PMs, each occupying a $30^{\circ}$ segment of the ring. The segments were mounted on the ID of a 4340 steel tube that had been banded with a filament-wound $\mathrm{M} 46 \mathrm{~J}$-carbon cylinder, which had an OD of $121 \mathrm{~mm}$ and a hoop strength of $2511 \mathrm{MPa}$. Before press fitting, the steel was hardened to a $1100 \mathrm{MPa}$ yield stress and the OD tapered at $0.88^{\circ}$ to a minimum of 105.2 $\mathrm{mm}$. Carbon band radial-compression failure is predicted at over $60,000 \mathrm{rpm}$, which is sufficient for prototypic applications. The PM segments were magnetized normal to their length, with the direction changed by $45^{\circ}$, from one segment to the next, in progressing around the ring.

After fabrication, the magnetic field was mapped inside, over a central 40-mm cubic region, immediately below, over a $15-\mathrm{mm}$ deep by $95-\mathrm{mm}$ square region, and outside the lower half of the band, in an annular region with an 152-mm OD. As expected from theory [9], for a very long magnet with a large number of magnet segments, the magnetic field in the interior was very unidirectional and normal to the cylindrical axis. Its magnitude was $0.17 \pm 0.03 \mathrm{~T}$. Generally, the unidirectional field was smallest along the central axis, becoming larger as the ID of the PMs were approached in the direction of magnetization, and smaller toward the ends of this short, non-ideal Halbach PM. The associated leakage from the ends produced a $<0.03 \mathrm{~T}$ field that will sweep through the HTS array with every turn of the rotor, like the beams of two back-to-back beacons. The associated idling losses will be large, compared to the HTS bearing losses. In longer rotors of prototypic design, the Halbach is away from the HTS.

The Halbach stator coil is contained in a vacuum-tight G10 cylinder, which was mounted in place of the electroturbine and clutch in Fig. 1. Motorized vertical positioning enabled its insertion, under vacuum, into the rotor's Halbach PM under vacuum. The air-cooled, iron-free stator winding was a 3-phase, wye-connected, 180-degree-pitch coil, with 75 turns of Litz wire, per phase. Three embedded Hall-effect switches provided position feedback to the drive electronics, which, in the motor mode, was a commercially available, 120-volt, brushless-DC servo amplifier configured in an "open loop". In the generate mode, the coil's 3-phase variable-voltage and variable-frequency sinusoidal waveforms were switched from the amplifier to a 3-phase diode-rectification bridge, whose variable DC output voltage was matched to load devices. The servo amplifier's, and the open-motor wiring's, drawback was radiated electrical noise that interfered with instrumentation. In preliminary spin testing, no idling losses were found for a similar open coil inserted in an earlier rotor's Halbach PM.

\section{Additional Lift Bearing}

The more massive B2 could not be levitated with the HTS bearing, alone. Although improvements in HTS processing continues, mass levitation capabilities of HTS bearings are limited. Prototypic size flywheels will require additional lift bearings. All permanent magnet bearings, or PM bearings, are well-suited for combination with HTS bearings. They contain passive elements and their magnetic-hysteretic drag torques during rotation can be less than those of HTS bearings, when similar PMs, with highly axial-symmetric magnetic fields, are used. Of course, PM bearings are statically unstable, thus the magnetic design must include stabilization with the HTS bearing. To levitate the larger mass of rotor B2, an Evershed-bearing configuration [10] was added.

In concept, the rotor is hung from the PM bearing, in axial attraction, and thrust upward with the HTS bearing, which, for stability, must have an absolute vertical stiffness that is larger. In practice, UGIMAG-34B PM rings were attached to the stator and the rotor, as in Fig. 3. Each had the same OD $(88.9-\mathrm{mm})$ and ID $(63.5-\mathrm{mm})$ and were either 6.4 or 12.7 $\mathrm{mm}$ thick. Reducing the gap between the rings of the PM bearing, increased the weight it supported and its negative vertical stiffness, which could be increased only to $<40$ $\mathrm{N} / \mathrm{mm}$, the HTS bearing's maximum. Since the bare PM's maximum speed was only $22,000 \mathrm{rpm}$, it was banded by press fitting a $102.5-\mathrm{mm}$ OD, AS4/Epon filament-wound ring over an E-glass ring, whose ID just fit over the OD of the PM ring. This increased the PM bearing's maximum speed to 55,000 rpm.

\section{TEST PROCEDURES AND RESULTS}

Each of the tests were performed similarly. Test set up involved positioning the rotor over the Cryohead to achieve: a level rotor, the desired uniform gap between the HTS PM and the Cryohead, and axial symmetry of the rotor with respect to both the Cryohead and the M/G spinup device. A motorized platform, which is not shown in Fig. 1, supported the weight of the rotor during field cooling and provided vertical positioning both before and during testing. Also, the platform provided both vertical and lateral confinement (high-speed touchdown ball bearings), a support for an additional PM bearing, and a base for the stator Coils.

After overnight establishment of a vacuum of $\sim 4 \mathrm{mPa}(30$ $\mu$ Torr), LN cooling began, which took several hours. Cryopumping decreased the pressure by another order of magnitude. Type- $\mathrm{K}$ thermocouples monitored the temperature of the Cryohead, in addition to any additional PMs and excitation coils. Then the support platform was lowered to achieve HTS levitation of the rotor and to set any additional PM bearing or stator Coil gaps. Spinup and spindown included several periods of coasting to measure idling losses.

As described before [2], knowing the mass properties of the rotor, a figure of merit for idling losses, the coefficient of friction, COF, is calculated using the spindown rate during coasting, which is measured using optical tachometers (Monarch ACT-3) that sense light/dark interfaces painted on the rotor. Also, the friction forces are assumed to occur at the mean radius of the HTS-PM rings, and zero-speed friction coefficients, $\mathrm{COF}_{0}$, were extrapolated. Non contact sensors (Kaman 2300) monitored the dynamic displacements of the rotor $O D$, after they were calibrated to the inductive properties of the carbon-filament bands.

Thirty-one tests were performed, including tests of both the HTS PMs, B1M and B2M, before their banding. Generally, during spinup, the peak radial motion was $\sim 1 \mathrm{~mm}$, below the rigid-body criticals, which reflects the eccentricity of the magnetic axis, with respect to the CG. Well above the criticals, the rotor self-centers and radial motion was 10 's of $\mu \mathrm{ms}$, which reflects the eccentricity of the centroidal axis 
with respect to the CG. Several mms of motion were typical in passing through the criticals. Also, during testing, resonances were detectable visually, with viewport TV cameras, and, while coasting, by the large rpm decay rates. In fact, speed-related variations in amplitudes and calculated COFs were similar.

As shown in Table 1, rotor B3 was spun to its speed limit, a rim velocity of $420 \mathrm{~m} / \mathrm{s}$, conservatively established to prevent HTS-PM radial crushing of their bands and loss of the Halbach $M / G$. The $M / G$ was capable of much higher speeds, which will be necessary for its use in the prototype. No rotor instablities were encountered here, nor with similar highly internally-damped, urethane-connected, rotors that were tested to $1100 \mathrm{~m} / \mathrm{s}$ rim speeds [7], on a quill shaft in a spinpit. Typically, intentional rotor-motion pertubations decayed.

An order of magnitude improvement in B3M's $\mathrm{COF}_{0}$, over B1M's, was found in tests performed at nearly the same gap, as shown in Table I. Improvement comes mainly from better processing of the individual HTS, which is also reflected in the average $\mathrm{F}_{0}$ measured for the $\mathrm{B} 3$ and $\mathrm{B} 1$ arrays reported in Table I. The levitation forces of individual HTS and speedindependent hysteretic losses of small-scale arrays are known to improve with increased critical current $\left(\mathrm{J}_{\mathrm{c}}\right)[2,6]$. A much smaller, but measured, part of the decrease in the COF was attributed to improved design of the B3 Cryohead, especially, its external insulation with layers of aluminized mylar film. Reduction in radiation losses decreases the operating temperature of the Cryohead by several degrees, thus increasing $\mathrm{J}_{\mathrm{c}}$.

As the speed of B1 and B2 were increased, with the ET $\mathrm{M} / \mathrm{G}$, the COFs increased to nearly constant values that depend, as shown in Fig. 4, on both the $\mathrm{COF}_{0}$ and the use of additional PM bearings. The great sensitivity of COF to the HTS-PM gap, and, thus, PM field inhomogeneities, is well known [2, 3] and seen in Fig. 4. For B2's smallest gap of 3.9 $\mathrm{mm}$, the high-speed COF increased to $2.4 \times 10^{-5}$, as reported in Table I. Less known, but proven [11], are the eddy-current losses created in the PM by nonuniform trapped-fields in the HTS. HTS trapped fields and eddy-current losses are made smaller (negligible) by reducing (eliminating) PM movement during levitation and field cooling of the HTS, which occurs as the PM bearing assumes more (all) of the rotor weight.

\section{CONCLUSIONS}

Technology for building and stably operating high-speed disk-shaped rotor flywheel systems has been demonstrated. HTS bearings and a Halbach $M / G$ are available for elastomeric connection in larger, prototypic (kW-hr) flywheel systems. Combining HTS and PM bearings, in Evershed configurations, promises to reduce losses and make diurnal energy storage an economically competitive option to expansion of energy production capacity.

\section{REFERENCES}

[1] R. Abboud, J. Hull, K. Uherka, and T. Mulcahy, "Flywheel energy storage using superconducting magnetic bearings," Proc. Am. Power Conf., vol. 56-II, pp. 1356-1361, A pril 1994.

[2] J. R. Hull, T. M. Mulcahy, K. L. Uherka, R. A. Erck, and R. A. Abboud. "Flywheel energy storage using superconducting magnetic bearings," Appl. Supercond., vol. 2, pp. 449-455, 1994.

[3] H. J. Borneman et al., "Low friction in a flywheel system with passive superconducting bearings," Appl. Supercond. vol. 2, pp. 439, 1994.
[4] T. M. Mulcahy et al., "A Permanent-MagnetRotor foraHigh-Temperature Superconducting Bearing," IEEE Trans. Magnetics," vol. 32, pp. 26092612 , July 1996

[5] J. R. Hull, "Flywheels on a roll," IEEE Spectrum, vol. 34, pp. 20-25, July 1997.

[6] V. R. Todt et al., "Processing of Large $\mathrm{YBa}_{2} \mathrm{Cu}_{3} \mathrm{O}_{\mathrm{x}}$ Domains for Levitation Applications by a Nd $1+x \mathrm{Ba}_{2-\mathrm{x}} \mathrm{Cu}_{3} \mathrm{O}_{y}$-Seeded Melt-Growth Technique "J. Electronic Materials.," vol. 23, pp. 1127-1130, 1994.

[7] C. W. Gabrys and C. E. Bakis, "Design and Testing of Composite Flywheel Rotors," in Composite Materials: Testing and Design, vol. 13, STP 1242, S. J. Hooper, Ed., Conshohocken, PA: American Society for Testing and Materials, 1997, pp. 3-22.

[8] W. L. Lorimer, D. K. Lieu, J. R. Hull, T. M. Mulcahy, and T. D. Rossing, "Correlation of Experimental Data and Three-Dimensional Finite Element Modeling of a Spinning Magnet Array," IEEE Trans. Magnetics., vol. 30, pp. 3003-3007, September 1994.

[9] R. F. Post, T. K. Fowler, and S. F. Post, "A High-Efficiency Electromechanical Battery," Proc. IEEE, vol. 81, pp. 462-474, March 1993.

[10] S. Whitely, "Review of the gas centrifuge until 1962: Principles of highspeed rotation," Rev. Mod. Phys., vol. 56, pp. 67-97, January 1984.

[11]...J. R. Hull, T. M. Mulcahy, and J. F. Labataille, "Velocity dependence of rotational loss in Evershed-Type superconducting bearings," Appl. Phys. Lett., vol. 70, pp. 655-657, February 3, 1997

TABLE I - ROTOR PROPERTIES AND RESULTS

\begin{tabular}{ccccc}
\hline Property & B1M(B3M) & B1 & B2 & B3 \\
\hline Mass $(\mathrm{kg})$ & 3.11 & 7.92 & 13.8 & 9.32 \\
I(kg-m $\left.{ }^{2}\right)$ & 0.022 & 0.115 & 0.486 & 0.131 \\
OD(m) & 0.203 & 0.394 & 0.590 & 0.394 \\
Aray F $_{0}(\mathrm{~N})$ & $10.2(18.7)$ & 10.2 & 15.6 & 18.7 \\
Gap $(\mathrm{mm})$ & $11.6(11.1)$ & $4.9(10.9)$ & $3.9(5.6)$ & 2.7 \\
M/G & ETSC & ETSC & ETSC(Coils) & Halbach \\
PM Bearing & No & No(Yes) & yes & No \\
Max. krpm & $2.22(2.56)$ & $11.9(5.40)$ & $9.01(9.77)$ & 20.4 \\
Rim V(m/s) & $23.6(27.2)$ & $245(112)$ & $278(302)$ & 420 \\
Max. KE(J) & $0.60(0.80)$ & $88.5(1.9)$ & $216(254)$ & 298 \\
COF $0 \times 10^{6}$ & $7.0(0.7)$ & $8.5(1.9)$ & $24(80)^{\mathrm{a}}$ & 4000 \\
\hline
\end{tabular}

${ }^{\text {a }}$ At zero speed, except at maximun speed for $B 2$

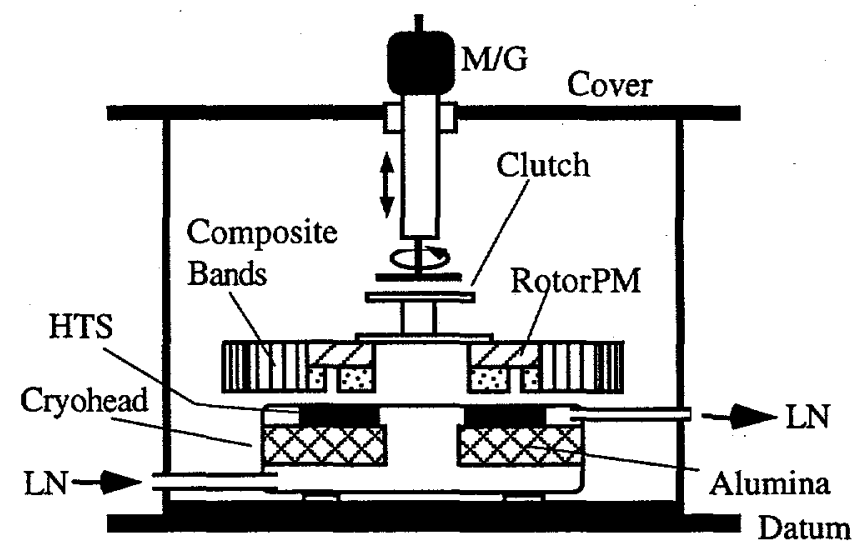

Fig. 1. Rotor $B 1$ in vacuum chamber, levitated over cryohead, with spinup by electroturbine and eddy current clutch (not to scale).

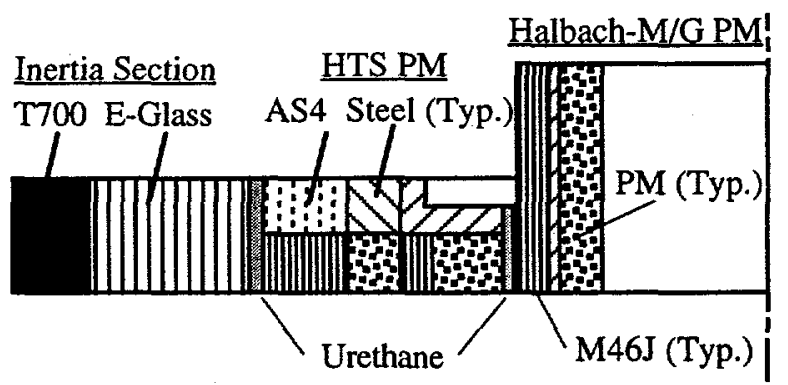

Fig. 2. Rotor B3 inertia section and Halbach-M/G PM connected to HTS PM by urethane filled annuli (not to scale). 


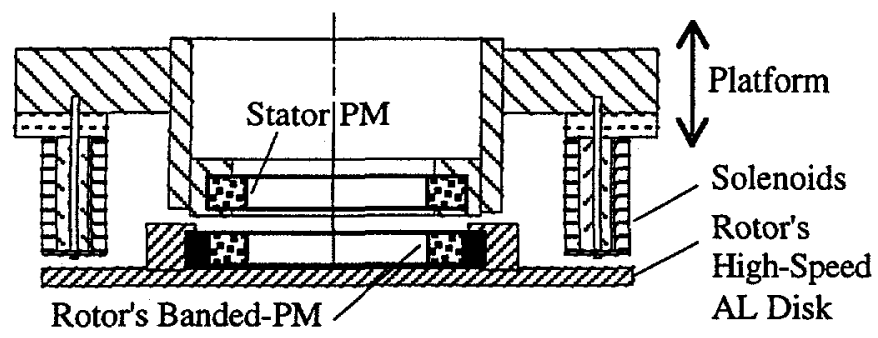

Fig. 3. Addtional PM bearing, and M/G Coil on motorized platform.

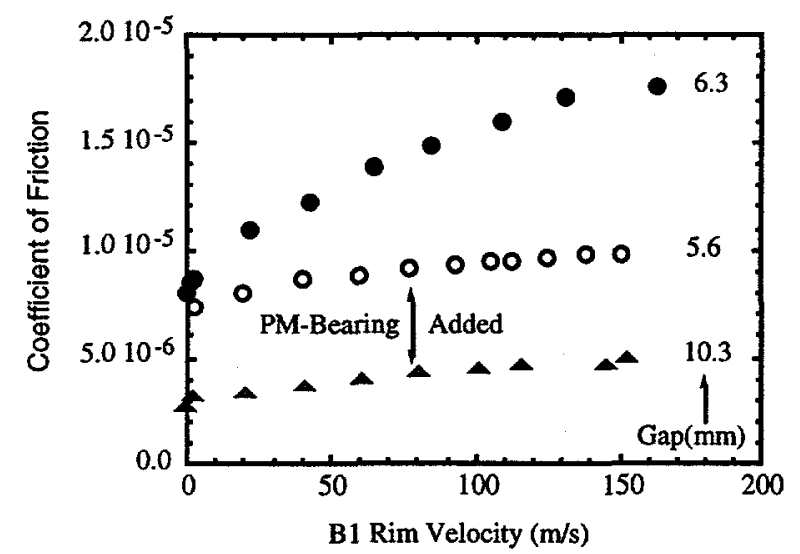

Fig. 4. High-speed COF nearly constant, especially with added PM bearing. 